Check for updates

Cite this: Chem. Sci., 2019, 10, 4313

๑ All publication charges for this article have been paid for by the Royal Society of Chemistry

Received 22nd November 2018

Accepted 8th March 2019

DOI: $10.1039 / c 8 s c 05218 b$

rsc.li/chemical-science

\title{
De novo synthesis of mesoporous photoactive titanium(Iv)-organic frameworks with MIL-100 topology $\dagger$
}

\author{
Javier Castells-Gil, (D) ${ }^{a}$ Natalia M. Padial, (D) ${ }^{\text {ab }}$ Neyvis Almora-Barrios, (DD ${ }^{a}$ Ivan da \\ Silva, (D) ${ }^{c}$ Diego Mateo, ${ }^{d}$ Josep Albero, ${ }^{d}$ Hermenegildo García (D) ${ }^{d}$ \\ and Carlos Martí-Gastaldo (iD) *a
}

\begin{abstract}
Most developments in the chemistry and applications of metal-organic frameworks (MOFs) have been made possible thanks to the value of reticular chemistry in guiding the unlimited combination of organic connectors and secondary building units (SBUs) into targeted architectures. However, the development of new titanium-frameworks still remains limited by the difficulties in controlling the formation of persistent Ti-SBUs with predetermined directionality amenable to the isoreticular approach. Here we report the synthesis of a mesoporous Ti-MOF displaying a MIL-100 topology. MIL-100(Ti) combines excellent chemical stability and mesoporosity, intrinsic to this archetypical family of porous materials, with photoactive $\mathrm{Ti}_{3}\left(\mu_{3}-\mathrm{O}\right)$ metal-oxo clusters. By using high-throughput synthetic methodologies, we have confirmed that the formation of this SBU is thermodynamically favored as it is not strictly dependent on the metal precursor of choice and can be regarded as an adequate building block to control the design of new Ti-MOF architectures. We are confident that the addition of a mesoporous solid to the small number of crystalline, porous titanium-frameworks available will be a valuable asset to accelerate the development of new porous photocatalysts without the pore size limitations currently imposed by the microporous materials available.
\end{abstract}

\section{Introduction}

Metal-organic frameworks (MOFs) are crystalline, porous materials built from the interlinking of metal-oxo clusters with organic connectors. ${ }^{1}$ Their internal porosity can be engineered in size, shape and chemical function to provide a family of crystalline solids with structural and functional diversities beyond comparison. These intrinsic characteristics make MOFs appealing materials for modern storage, separation, delivery and catalysis technologies. ${ }^{2-6}$ The problems linked to the development of these applications have stressed the need for producing MOFs with superior chemical stability. ${ }^{7}$ Combination of hydrolytic stability with high surface area was first reported for sodalite-type frameworks built from imidazolate

${ }^{a}$ Universidad de Valencia (ICMol), Catedrático José Beltrán-2, 46980, Paterna, Spain. E-mail: carlos.marti@uv.es

${ }^{b}$ Department of Chemistry, The Scripps Research Institute, 10550 North Torrey Pines Road, La Jolla, California, 92037, USA

'ISIS Facility, Rutherford Appleton Laboratory, Chilton, Didcot, Oxfordshire, OX11 $O Q X, U K$

${ }^{d}$ Instituto Universitario de Tecnología Química CSIC-UPV, Universitat Politècnica de València, Av. De Los Naranjos s/n, 46022, Valencia, Spain

$\dagger$ Electronic supplementary information (ESI) available. CCDC 1871195. For ESI and crystallographic data in CIF or other electronic format see DOI: $10.1039 / \mathrm{c} 8 \mathrm{sc} 05218 \mathrm{~b}$
$(\mathrm{ZIF})^{8}$ or azolate ${ }^{9}$ connectors due to hydrophobic shielding and strong metal-nitrogen bonds. Control over the strength of the coordination bond is also key to the robustness of $\mathrm{Zr}(\mathrm{IV})$ and Hf(Iv) MOFs.

Compared to these metals, titanium offers several advantages. It is less toxic, naturally more abundant and more adequate to endow these porous materials with photoactivity. Still, the synthesis of crystalline, porous titanium-organic frameworks remains comparatively underexplored..$^{10}$ Though the discovery of the UiO family, based on $\mathrm{Zr}_{6} \mathrm{O}_{4}(\mathrm{OH})_{4}\left(\mathrm{RCO}_{2}\right)_{12}$ clusters, ${ }^{11}$ boosted the synthesis of multiple MOFs from isostructural $\mathrm{Zr}$ and $\mathrm{Hf}$ metal-oxo clusters, ${ }^{12-14}$ the controllable synthesis of Ti-MOFs remains quite challenging yet. This is due to the higher reactivity of Ti precursors, more prone to hydrolysis, that facilitates the formation of amorphous titanium oxide under solvothermal conditions. The formation of titanium-oxocarboxylate clusters, capable of directing the formation of high symmetry, periodic architectures, demands stricter control over the hydrolysis-condensation equilibria. These synthetic difficulties are possibly the reason due to which almost all Ti-MOFs available are built from different secondary building units (SBUs). Fig. 1 summarizes the diversity of Ti-SBUs reported for the limited number of MOFs available: $\mathrm{Ti}_{12} \mathrm{O}_{15} / \mathrm{Ti}_{6} \mathrm{O}_{9}$ titaniumoxo clusters in MIL-177, ${ }^{15} \mathrm{Ti}_{8} \mathrm{O}_{8}$ in MIL- $125,{ }^{16} \mathrm{Ti}_{7} \mathrm{O}_{4}$ in PCN-22, ${ }^{17}$ $\mathrm{Ti}_{6} \mathrm{O}_{6}$ in MOF-901 (ref. 18) and MTM- $1,{ }^{19} \mathrm{Ti}_{3} \mathrm{O}$ in COK-69, ${ }^{20}$ 

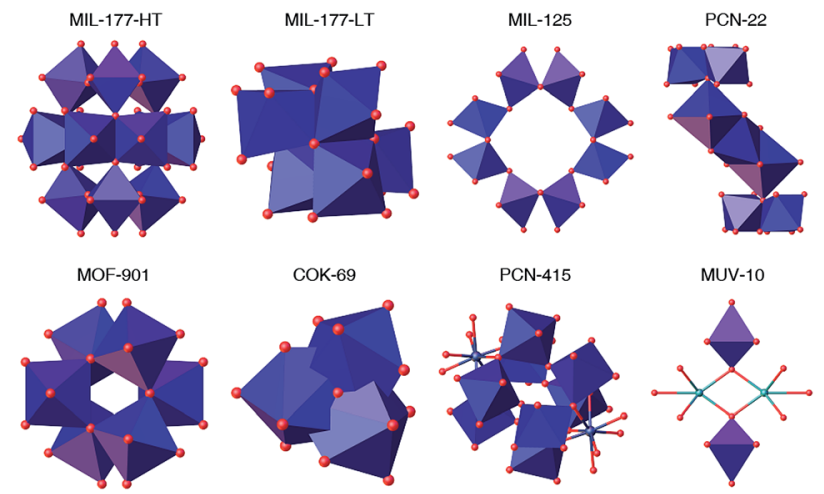

Fig. 1 Summary of the SBUs in the structure of titanium-organic frameworks.

heterometallic clusters like $\mathrm{Ti}_{8} \mathrm{Zr}_{2} \mathrm{O}_{12}$ in PCN-415 (ref. 21) and $\mathrm{Ti}_{2} \mathrm{M}_{2} \mathrm{O}_{2}(\mathrm{M}=\mathrm{Ca}, \mathrm{Mn})$ in MUV-10, ${ }^{22}$ or simple titanium nodes in NTU-9 (ref. 23) and Ti-CAT-5 ${ }^{24}$ confirm the complexity for targeting the formation of specific MOF architectures due to the difficulties in controlling the formation of persistent SBUs.

This lack of chemical control over the final material is arguably limiting further development, highlighting the importance of finding alternative synthetic strategies that make Ti-MOFs amenable to the design principles of isoreticular chemistry. One strategy used to gain control is to produce daughter frameworks by incorporation of titanium(Iv) into preformed materials by partial post-synthetic replacement of parent metals. This was originally demonstrated for the UiO66 $(\mathrm{Zr})^{25}$ and MOF-5(Zn $)^{26}$ families. More recent examples describe the use of titanium(III) precursors followed by mild oxidation for partial doping of MOF-74, MIL-100 and PCN-333 with $\mathrm{Ti}^{27}$ However, these methodologies do not allow for complete metal replacement and also suffer from poor control over the positioning of titanium atoms in the structure, ${ }^{28}$ which can result in spurious deposition of metal oxide coatings. ${ }^{29}$

Compared to this post-synthetic strategy, here we report the formation of MIL-100(Ti) by direct synthesis. Systematic exploration of the chemical space, by using high-throughput (HT) methodologies, has enabled identification of the optimal synthetic conditions to produce highly crystalline solids from different titanium precursors. MIL-100(Ti) combines the chemical stability and mesoporosity, intrinsic to the MIL-100 family, with photoactive $\mathrm{Ti}_{3}\left(\mu_{3}-\mathrm{O}\right)$ metal-oxo clusters. Our work suggests that the formation of this SBU, classical in the chemistry of MOFs based on trivalent metals, is not strictly dependent on the metal source and might be used for the controlled design of new Ti-MOF architectures.

\section{Results and discussion}

\section{Synthesis from pre-formed metal clusters}

Our first approach to the direct synthesis of Ti-MOFs involved the use of titanium-oxo clusters as pre-formed SBUs. In principle, this was expected to favour the formation of crystalline solids under milder conditions than those conventionally used in MOF synthesis. This strategy has been successfully used to produce $\mathrm{Zr}(\mathrm{IV}),{ }^{30} \mathrm{Ce}(\mathrm{Iv}),{ }^{31}$ and $\mathrm{Fe}(\mathrm{III})-\mathrm{MOFs}^{32}$ by direct reaction of metal-oxo clusters with polyaromatic carboxylic acids. Compared to simple precursors, we also expected to minimize the precipitation of amorphous oxides due to the higher stability of their cluster against hydrolysis. Accordingly, we reacted the as-made $\left[\mathrm{Ti}_{6} \mathrm{O}_{6}(4-\mathrm{tbbz})_{6}\left(\mathrm{O}^{\mathrm{i}} \mathrm{Pr}\right)_{6}\right]($ tbbz $=4$-tert-butylbenzoic acid) clusters (Fig. 2a) with trimesic acid $\left(\mathrm{H}_{3}\right.$ btc $=$ benzene-1,3,5-tricarboxylic acid) between 80 and $160{ }^{\circ} \mathrm{C}$ for multiple combinations of solvents (DMF, DEF, NMP, MeOH, EtOH...), metal to linker ratios, concentration and addition of different modulators like acetic or benzoic acid. We used a FLEX SHAKE high-throughput workstation from Chemspeed( $\odot$ for robotic dispensing of solids and liquids in order to optimize the screening of a broad range of synthetic conditions whilst ensuring reproducibility. All attempts were unsuccessful as we could only isolate the unreacted cluster or amorphous solids for temperatures below or above $100{ }^{\circ} \mathrm{C}$, respectively. We argued this was due to the poor solubility of the cluster under these conditions and decided to investigate an alternative mixture of solvents. After several tests, we observed that heating of a mixture of $\left[\mathrm{Ti}_{6} \mathrm{O}_{6}(4-\mathrm{tbbz})_{6}\left(\mathrm{O}^{\mathrm{i}} \mathrm{Pr}\right)_{6}\right]\left(\mathrm{Ti}_{6}\right)$ and $\mathrm{H}_{3}$ btc in acetonitrile : tetrahydrofuran (ACN : THF, $3: 1 \mathrm{v} / \mathrm{v} \%$ ) led to the formation of a white, microcrystalline solid. In order to optimize the crystallinity of the material we systematically varied the temperature of the reaction, the addition of acetic acid as the modulator, and the metal-to-linker ratio. We observed that, for a given $\mathrm{Ti}$ : btc ratio of $1: 5$, only the unreacted cluster was obtained at temperatures below $140{ }^{\circ} \mathrm{C}$ with no addition of the modulator (Fig. S1-S4†). However, a crystalline solid is observed in the presence of acetic acid as the modulator $(180-550$ equiv., 250-750 $\mu \mathrm{L}$ ) and at temperatures above $100{ }^{\circ} \mathrm{C}$. Note that a secondary crystalline phase is obtained as a side-product at low temperatures and high amounts of acetic acid (i.e. 550 equiv., $750 \mu \mathrm{L}$ ) as evidenced by powder X-ray diffraction (PXRD) (Fig. S2-S5†), whereas it is not observed for a lower concentration of the modulator. The formation of this secondary phase can be reduced by increasing the temperature of the reaction up to $160^{\circ} \mathrm{C}$ (Fig. $2 \mathrm{~b}-\mathrm{c}, \mathrm{S} 6$ and $\mathrm{S} 7 \dagger$ ). We next varied the $\mathrm{Ti}$ : btc ratio at $160{ }^{\circ} \mathrm{C}$ for a fixed amount of acetic acid (i.e. 180 equiv., 250 $\mu \mathrm{L})$. PXRD of the solids confirms the formation of a highly crystalline MIL-100 phase for Ti : btc ratios of $1: 2-5$ (Fig. S8†). PXRD of the solids synthesized with increasing temperatures and concentration of the modulator confirms the critical effect of these parameters on the crystallinity of the material (Fig. 2b and c). The optimum conditions for the formation of highly crystalline MIL-100(Ti) corresponds to a Ti : btc ratio of $1: 5$ and addition of $250 \mu \mathrm{L}$ of acetic acid (180 equiv.) and reaction at $160{ }^{\circ} \mathrm{C}$ (see $\mathrm{ESI} \dagger$ for details). The use of more conventional solvents like DMF or DEF yielded amorphous or poorly crystalline materials under equivalent reaction conditions (Fig. S9 and S10 $\dagger$ ). Optical microscopy suggested the formation of small crystals, which was confirmed by scanning electron microscopy (SEM). As shown in Fig. 2e, the bulk is composed of single crystals with an octahedral habit, typical of cubic MIL-100 phases, and sizes oscillating below $5 \mu \mathrm{m}$. We also identified small fractions of amorphous material partially covering the 

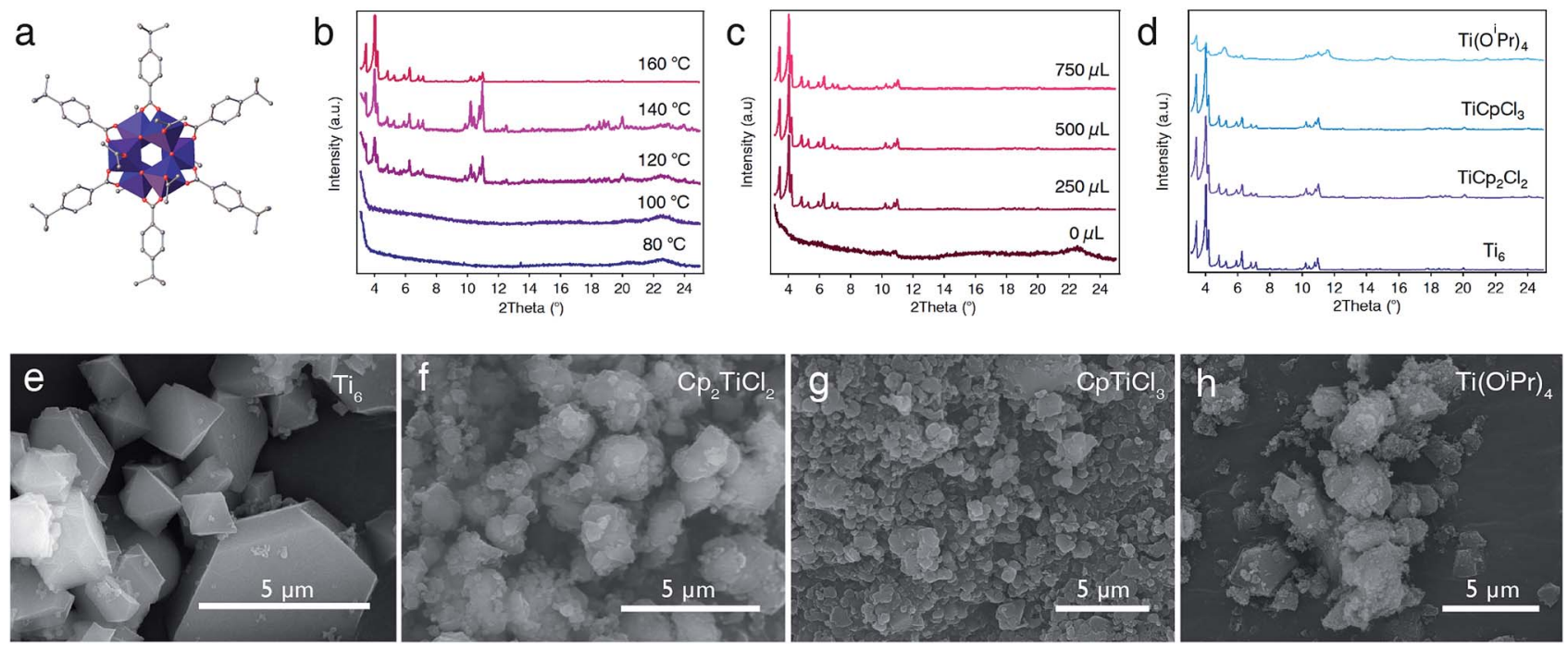

Fig. 2 Optimization of reaction conditions for different titanium precursors to form MIL-100(Ti). (a) Structure of the titanium-oxo cluster $\left[\mathrm{Ti}_{6} \mathrm{O}_{6}(4-\mathrm{tbbz})_{6}\left(\mathrm{O}^{\mathrm{P} P r}\right)_{6}\right]\left(\mathrm{Ti}_{6}\right)$ used as the metal source. PXRD of the materials formed under different conditions showing the effect of (b) temperature, (c) amount of modulator at $160^{\circ} \mathrm{C}$ and (d) Ti(IV) precursor used in the synthesis on the crystallinity of the formed phase. (e-h) SEM micrographs of the solid obtained with each Ti(Iv) precursor showing the formation of micrometric, octahedral single crystals for Ti 6 .

surface of the crystals (Fig. S11†). The solid was recovered by centrifugation and washed with DMF and $\mathrm{MeOH}$ prior to drying in a vacuum at room temperature.

\section{Structure and characterization}

PXRD patterns of the solid collected at our laboratory revealed clear similarities to the reported phases of MIL-100(Cr, Fe) (Fig. S16 $\dagger$ ). ${ }^{33,34}$ For a clearer structural description, we collected high-resolution PXRD data that were refined by using an starting model built from the reported structure of MIL-100(Fe) by using Materials Studio. As shown in Fig. 3a, Rietveld refinement of the activated solid converged with excellent residual values $\left(R_{\mathrm{wp}}=5.24 \%, R_{\exp }=1.93 \%\right)$ for a cubic $F d \overline{3} m$ space group with cell parameter $a=73.5168(16) \AA$. The structure of MIL-100(Ti) is built from the interlinking of $\mathrm{Ti}_{3}\left(\mu_{3}-\mathrm{O}\right)$ clusters and btc linkers to conform a zeolitic mnt topology that features two types of mesoporous cages with pentagonal and hexagonal pore windows (Fig. 3b).

The assembly of this structure from the starting $\mathrm{Ti}_{6} \mathrm{O}_{6}$ cluster used in the synthesis necessarily involves its degradation for the assembly of a thermodynamically more stable unit under the reaction conditions. This dynamic equilibrium between interconverting species is similar to that controlling the formation of heptameric clusters or Ti-oxo chains from $\mathrm{Ti}_{6} \mathrm{O}_{6}(\mathrm{abz})_{6}\left(\mathrm{O}^{\mathrm{i}} \mathrm{Pr}\right)_{6}$ ( $\mathrm{abz}=4$-aminobenzoate) during the synthesis of PCN-22 (ref. 17) or DGIST $-1,{ }^{35}$ respectively. By using comparatively milder conditions, the integrity of the starting homo or heterometallic polynuclear Ti(Iv) clusters can also be preserved and they can be incorporated into the framework, as demonstrated for MOF901, ${ }^{18}$ MNT-1 (ref. 19) or PCN-405. ${ }^{21}$ Compared to other precursors, these precedents seem to confirm the suitability of robust, molecular Ti clusters to either retain their nuclearity or control the formation of alternative SBUs more prone to induce the formation of specific MOF architectures under thermodynamic control. For example, $\mathrm{M}_{3}\left(\mu_{3}-\mathrm{O}\right)(\mathrm{M}=\mathrm{Cr}, \mathrm{Al}, \mathrm{Fe})$ SBUs are very common in frameworks produced under relatively harsh conditions like MIL-96 (ref. 36) or MIL-101. ${ }^{37}$

The replacement of trivalent metals with Ti(rv) is not directly compatible with the local structure of MIL-100 materials, which features 6-connected $\left[\mathrm{M}_{3}^{\mathrm{III}}\left(\mu_{3}-\mathrm{O}\right)(\mathrm{X})\left(\mathrm{H}_{2} \mathrm{O}\right)_{2}(\mathrm{btc})_{6}\right]\left(\mathrm{X}^{-}=\mathrm{F}, \mathrm{Cl}, \mathrm{OH}\right)$ nodes. In our case, the introduction of an excess of one positive charge per metal atom will be counterbalanced by additional anions. We hypothesized that these could be directly coordinated to the Ti sites or occupy the empty space available from the mesoporous cages for neutral or cationic frameworks, respectively. FT-IR spectra of the solid, before and after Soxhlet washing (Fig. S19†), ${ }^{1} \mathrm{H}-\mathrm{NMR}$ (Fig. S26 $\dagger$ ) and solid-state ${ }^{13} \mathrm{C}$-CP-MAS-NMR (Fig. S28 $\dagger$ ), all discard the presence of $\mathrm{Ti}_{6}$ clusters or free trimesate or acetate anions occupying the pores. Hence, we modelled computationally different neutral clusters by modification of the molecules coordinated to the axial position of the octahedral Ti(Iv) centers whilst fixing the positions corresponding to the btc linkers and the $\mu_{3}-\mathrm{O}$ central bridge. We used the same methodology recently reported by De Vos and co-workers for rationalizing the most plausible formula of COK-69, ${ }^{20}$ based on analogous Ti(rv)-oxo trimers. We calculated the formation energies for the clusters that would generate a neutral structure by different combinations of $\mathrm{O}^{2-}, \mathrm{OH}^{-}$or $\mathrm{H}_{2} \mathrm{O}$ molecules acting as capping linkers. We optimized their structure with dispersion-corrected density functional theory (DFT-D3) as implemented in the Vienna ab initio simulation package (VASP) (see $\mathrm{S} 5 \uparrow$ for calculation details). Our results suggests that the formation of the cluster $\left[\mathrm{Ti}_{3}\left(\mu_{3}-\mathrm{O}\right) \mathrm{O}(\mathrm{OH})_{2}(\mathrm{btc})_{6}\right]$ (Fig. 3c, left) is $0.21 \mathrm{eV}$ more favourable than $\left[\mathrm{Ti}_{3}\left(\mu_{3}-\mathrm{O}\right)(\mathrm{O})_{2}\left(\mathrm{H}_{2}-\right.\right.$ O)(btc) $)_{6}$ (Fig. 3c, right). Nonetheless, like in the case of COK-69, the small difference between both clusters (c.f. $0.4 \mathrm{eV}$ ) is not sufficient to confirm unambiguously the formation of only one of them and both formulae might be reasonable. 


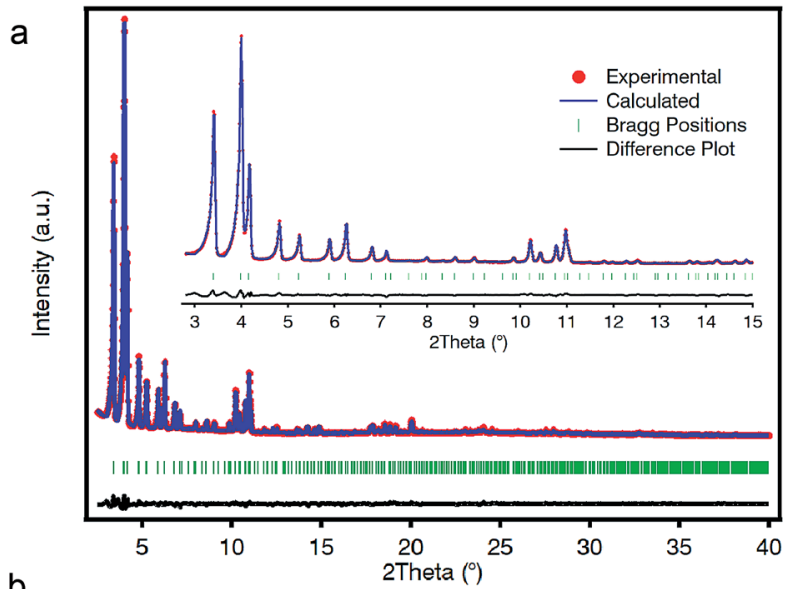

b

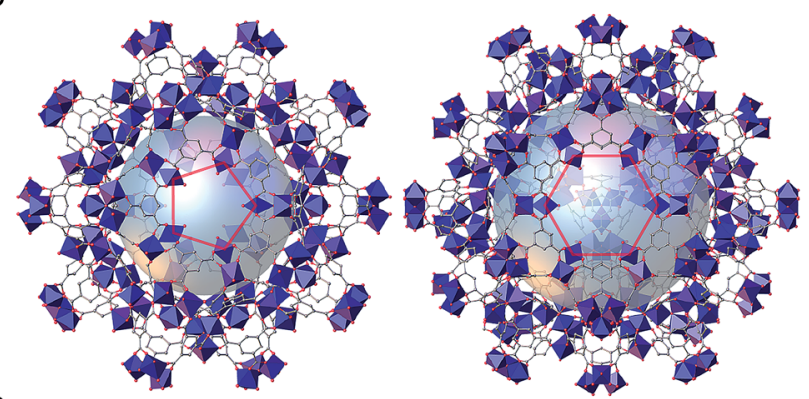

C
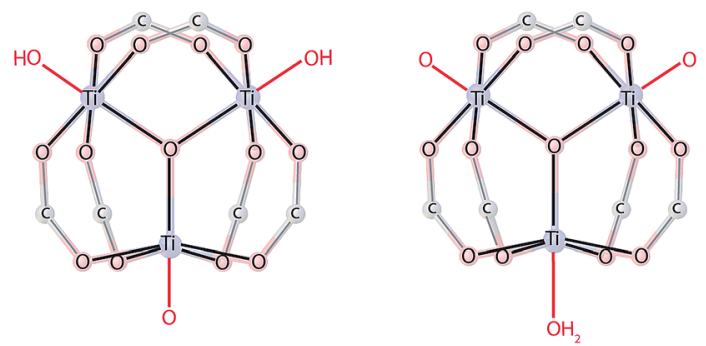

Fig. 3 Structural characterization of MIL-100(Ti). (a) Rietveld refinement of MIL-100(Ti) ( $\left.\lambda_{\mathrm{CuK}_{\alpha 1}}=1.540598 \AA, \lambda_{\mathrm{CuK}_{\alpha 2}}=1.544426 \AA\right)$. See SI3 for crystallographic data and full details. (b) Perspective of the structure along the [001] and [111] showing the presence of two types of mesoporous cages ( 2.4 and $2.9 \mathrm{~nm}$ ) with pentagonal and hexagonal windows intrinsic to the MIL-100 family. (c) Proposed structure of the more favorable Ti-SBUs compatible with the formation of neutral MIL100 (Ti) featuring $\mathrm{OH}^{-} / \mathrm{O}^{2-}$ (left) or $\mathrm{O}^{2-} / \mathrm{H}_{2} \mathrm{O}$ (right) capping ligands.

The proposed formulae agree well with the percentage of residual oxide formed from the thermal decomposition of the solid (Fig. S21†). The experimental $32.2 \%$ of $\mathrm{TiO}_{2}$ is quite close to the calculated value of $33.9 \%$. Permanent porosity was studied using $\mathrm{N}_{2}$ adsorption-desorption isotherms at $77 \mathrm{~K}$ after activation of the solid under dynamic vacuum $\left(10^{-3} \mathrm{mbar}\right)$ at $150{ }^{\circ} \mathrm{C}$ overnight. MIL-100(Ti) displays a non-hysteretic, type-I $\mathrm{N}_{2}$ adsorption with two secondary gas uptakes at $P / P_{0}=0.05$ and 0.14 (Fig. 4a). The multi-point BET surface area was found to be $1321 \mathrm{~m}^{2} \mathrm{~g}^{-1}$ with a total pore volume of $0.66 \mathrm{~cm}^{3} \mathrm{~g}^{-1}$ (Fig. S22 and Table S3†). Pore size distribution (PSD) calculated using Non-Linear Density Functional Theory (NLDFT) models reveals two types of mesoporous pores between 1.6-2.4 and 2.6$3.6 \mathrm{~nm}$ that correlate well with the theoretical pore size values of
2.4 and $2.9 \mathrm{~nm}$ estimated from the structure of MIL-100(Ti). This surface area lies below the values reported for MIL$100(\mathrm{Cr}, \mathrm{Fe})$ phases, ${ }^{33,34}$ suggesting ineffective activation or the partial blocking of the pores by molecular precursors with poor solubility. We used other activation protocols more adequate for highly porous materials like solvothermal treatment of the solid or Soxhlet washing with hot EtOH or $\mathrm{MeOH}$. None of them led to a substantial increase in porosity. As commented before, FT-IR, ${ }^{1} \mathrm{H}-\mathrm{NMR}$ and ${ }^{13} \mathrm{C}-\mathrm{CP}-\mathrm{MAS}-\mathrm{NMR}$ of the activated solid rule out the presence of molecular components occluded in the pores of MIL-100(Ti). Thus, we ascribe the reduction of surface area to the formation of an amorphous fraction of the material that can be inferred from SEM (Fig. S11†), similar to previous reports for other mesoporous phases like MIL-101-NDC(Cr). ${ }^{38}$

$\mathrm{CO}_{2}$ adsorption studies reveal a modest uptake of $1.70 \mathrm{mmol}$ $\mathrm{g}^{-1}(7.5 \mathrm{wt} \%)$ at $293 \mathrm{~K}$ and 1 bar, with an isosteric heat of adsorption $\left(Q_{\text {st }}\right)$ of $34.8 \mathrm{~kJ} \mathrm{~mol}^{-1}$ (Fig. $4 \mathrm{~b}$ and $\left.\mathrm{S} 24 \dagger\right)$. This value is very similar to the $30-35 \mathrm{~kJ} \mathrm{~mol}^{-1}$ reported for the $\mathrm{Fe}(\mathrm{III})$ phase $^{39}$ and agrees well with the presence of vacant metal sites. Water adsorption also reveals clear similarities with other MIL-100 derivatives. As shown in Fig. 4c, the isotherm at $298 \mathrm{~K}$ shows an 'S'-shaped profile with hysteretic behaviour, indicative of irreversible capillary condensation. Water adsorption at low relative pressures, which can be ascribed to the presence of open metal sites in the structure, is followed by a steep uptake at a relative humidity between 0.25 and $0.45 \%$ with an inflection point at $P / P_{0}=0.32$, characteristic of hydrophilic materials. ${ }^{40}$ The maximum water adsorption capacity of MIL-100(Ti) calculated at $P / P_{0}=0.9$ is $0.52 \mathrm{~g} \mathrm{~g}^{-1}$. This gravimetric adsorption is very similar to the value reported for MIL-100(Al) $\left(0.51 \mathrm{~g} \mathrm{~g}^{-1}\right)$ but smaller than those for the $\mathrm{Cr}$ and $\mathrm{Fe}$ phases ranging from 0.60 to $0.76 \mathrm{~g} \mathrm{~g}^{-1}$ (Fig. $4 \mathrm{c}$ and Table S4†). ${ }^{\mathbf{4 1 , 4 2}}$

\section{Thermal and chemical stability}

One of the main features of MIL-100 materials is their stability in water. For Fe, $\mathrm{Al}$ and Sc phases, this stability is thermodynamic in origin and arises from strong coordination bonds with $\mathrm{M}-\mathrm{O}$ bond dissociation energies of 444,502 and $671 \mathrm{~kJ} \mathrm{~mol}^{-1} \cdot{ }^{43}$ In turn, the kinetic inertness of $\mathrm{Cr}(\mathrm{III})$ ions in MIL-100(Cr) is responsible for its outstanding hydrolytic stability. Accordingly, the incorporation of highly charged Ti(Iv) metals into MIL-100 topologies will result in high thermal and chemical stability due to thermodynamic parameters. Compared to the $\mathrm{Cr}$ and $\mathrm{Fe}$ phases that decompose between 300 and $350{ }^{\circ} \mathrm{C},{ }^{33,34}$ the thermogravimetric analysis (TGA) of MIL-100(Ti) confirms a significant increase in the thermal stability of the solid that remains stable up to $450{ }^{\circ} \mathrm{C}$ (Fig. S21†). This thermal stability is similar to other Ti-MOFs and agrees well with the higher dissociation energy of O-Ti(Iv) bonds of $667 \mathrm{~kJ} \mathrm{~mol}^{-1}$. $^{43}$ We next evaluated the hydrolytic stability of MIL-100(Ti) by soaking freshly made solids in water under acidic and basic conditions ( $\mathrm{pH}$ 1-14). PXRD of the solids after incubation in water for 24 hours confirms that the structure of the solid remains unchanged between $\mathrm{pH} 2$ and 12 (Fig. 4d and $\mathrm{S} 32 \dagger)$. Stability was also confirmed by gas sorption. $\mathrm{N}_{2}$ adsorption measurements of the solids after water treatment show negligible changes in their surface area compared to the as-made material 
a

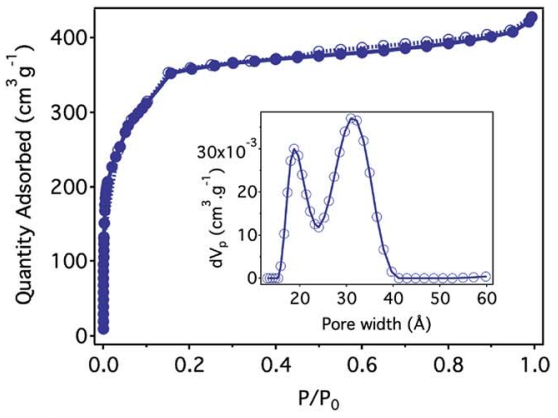

d

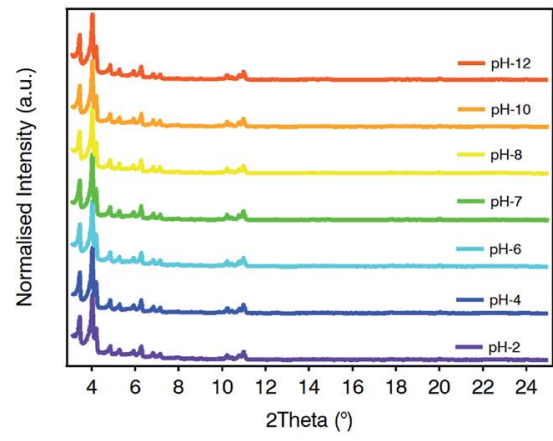

b

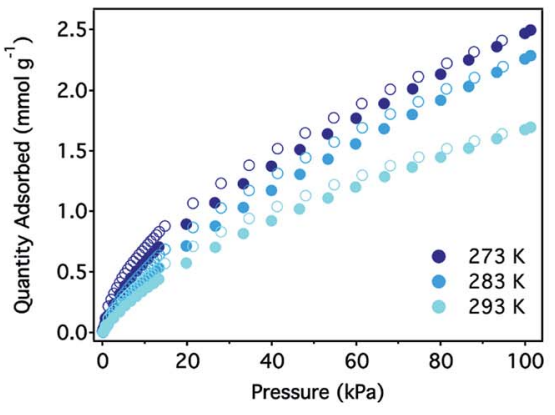

e

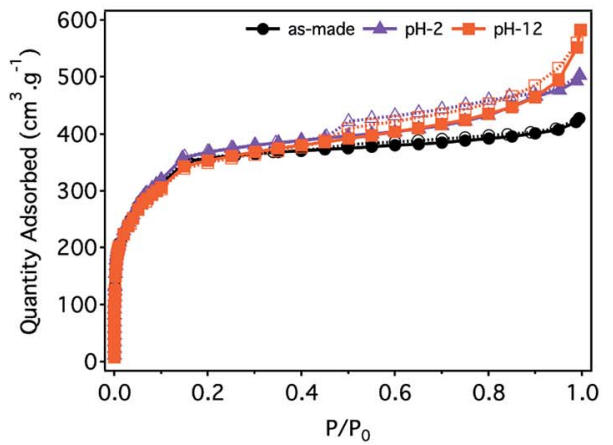

C

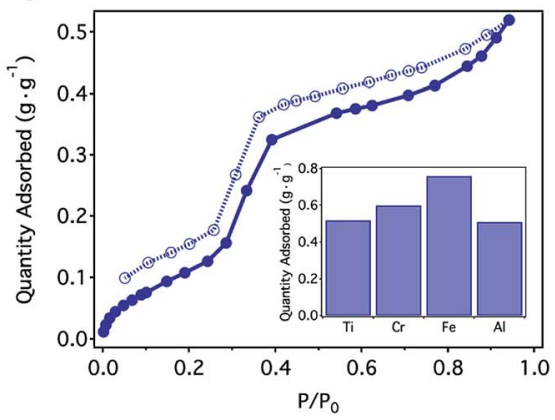

f

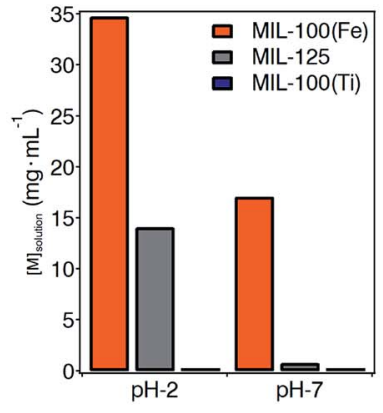

Fig. 4 Sorption properties and chemical stability of MIL-100(Ti). (a) $\mathrm{N}_{2}$ isotherm at $77 \mathrm{~K}$ and PSD (inset). (b) $\mathrm{CO}_{2}$ isotherms obtained at 273,283 and $293 \mathrm{~K}$. (c) Water isotherm at $298 \mathrm{~K}$ and comparison of the total water adsorption at $P / P_{0}=0.9$ with the isostructural $\mathrm{Cr}$, Fe and Al MIL-100 phases (inset). (d) PXRD of the solid after soaked in water for 24 hours at variable $\mathrm{pH}$. (e) Comparison of the $\mathrm{N}_{2}$ adsorption isotherms at $77 \mathrm{~K}$ of the as-synthesized MIL-100(Ti) and after water treatment at pH 2 and 12. (f) ICP-MS measurements for MIL-100(Fe), MIL-125 and MIL-100(Ti) at acidic and neutral $\mathrm{pH}$.

(Fig. 4e, S33 and Table S5†). These results confirm that MIL100(Ti) displays excellent hydrolytic stability, comparable to other benchmark materials. ${ }^{7}$

For further understanding on the role of $\mathrm{Ti}_{3}\left(\mu_{3}-\mathrm{O}\right)$ nodes over the chemical stability of the material we decided to carry out equivalent experiments for direct comparison with the isostructural MIL-100(Fe ${ }^{44}$ and a representative Ti-MOF like MIL$125 .{ }^{16}$ PXRD and $\mathrm{N}_{2}$ sorption confirm that replacement of $\mathrm{Fe}(\mathrm{III})$ with $\mathrm{Ti}(\mathrm{Iv})$ centers does not induce important changes under equivalent conditions. MIL-100(Fe) only shows minor degradation of the structure at $\mathrm{pH} 12$ (Fig. S34 $\dagger$ ), whereas its surface area is maintained close to the original value under acidic and basic conditions (Fig. S35 and Table S6 $\dagger$ ). This behaviour is different for MIL-125 that retains its structural integrity and porous function almost intact at $\mathrm{pH}-12$ but undergoes significant degradation at acidic pH (Fig. S36, S37 and Table S7†). We observe more important differences in the ICP-MS measurements of the supernatants after incubation in water. As shown in Fig. 4f, titanium leaching in MIL-100(Ti) is almost negligible for acidic and neutral conditions. In turn, the concentration of iron and titanium in solution increases with decreasing $\mathrm{pH}$ up to a maximum of 35 and $14 \mathrm{mg} \mathrm{mL}^{-1}$ at $\mathrm{pH} 2$ for MIL-100(Fe) and MIL-125, respectively (see Table S8 $\dagger$ for details). Concerning the isostructural series, we presume that the different degradation rates of the $\mathrm{Ti}$ and $\mathrm{Fe}$ phases might result in different drug delivery kinetics under simulated biological conditions. Although this study is beyond the scope of our work, we are confident that MIL-100(Ti) might be of interest to the MOF community for biomedical applications.

\section{Optical response and photoactivity}

The applications of Ti-MOFs as photocatalysts for solar fuel production or light-induced organic transformations are pivotal to this family of materials due to the promising combination of high surface areas, chemical stability and photoactivity. ${ }^{45}$ The optical band-gap of MIL-100(Ti) obtained by diffuse reflectance spectroscopy is $3.40 \mathrm{eV}$ (Fig. S30†). This value lies between those reported for MIL-125 $(3.68 \mathrm{eV})^{46}$ or MUV-10(Ca) $(3.10 \mathrm{eV})^{22}$ and is compatible with UV light photoexcitation. This was confirmed by irradiating a suspension of the solid in dry THF with UV-B light $(\lambda=280-315 \mathrm{~nm})$. After a few hours, the color of the solid changed from white to dark blue (Fig. S31 $\dagger$ ) and remained stable in the absence of oxygen. The electron paramagnetic resonance (EPR) spectra of the solid before/after irradiation confirms the occurrence of a broad signal at $350 \mathrm{mT}$, with $\mathrm{g}$ fitted parameters $g_{\|}=1.927$ and $g_{\perp}=1.952$ characteristic of Ti(III) species, only for the irradiated sample (Fig. 5a and S31). This is accompanied by a weak, sharp peak at lower fields, which corresponds to the formation of btc radicals with $g \approx$ $2.00 .^{22}$ This behaviour is consistent with a ligand-to-metal charge transfer (LMCT) mechanism, in which an electron is transferred from the photoexcited state of the linker to the metal sites (Fig. 5b). 

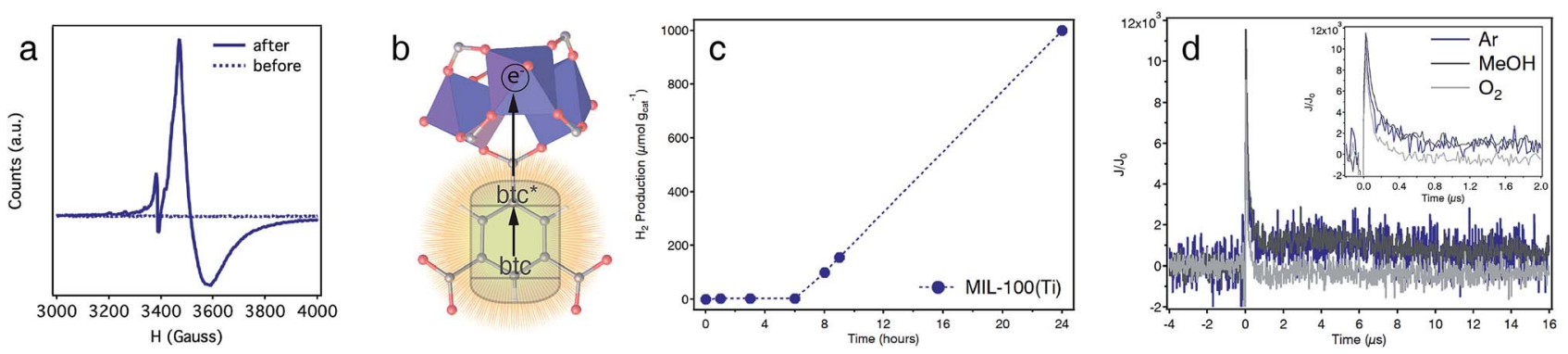

Fig. 5 Photoactivity of MIL-100(Ti). (a) EPR of MIL-100(Ti) before and after irradiation with UV-light. (b) Schematic showing the ligand-to-metal charge transfer responsible for the photogeneration of Ti(III) species. (c) Photocatalytic $\mathrm{H}_{2}$ production of $\mathrm{MIL}-100$ (Ti) in a mixture of $\mathrm{H}_{2} \mathrm{O}: \mathrm{MeOH}$ (4:1, v/v\%). (d) Transient absorption kinetics of MIL-100(Ti) suspended in ACN under Ar(blue), in the presence of MeOH (dark grey) and in the presence of $\mathrm{O}_{2}$ (light grey). Laser excitation: $355 \mathrm{~nm}$, monitored at $500 \mathrm{~nm}$. The inset shows a zoom-in of the first $2 \mu \mathrm{s}$.

We also tested the photocatalytic activity of MIL-100(Ti) by irradiating a suspension of the solid in a mixture of $\mathrm{H}_{2}-$ $\mathrm{O}: \mathrm{MeOH}(4: 1, \mathrm{v} / \mathrm{v} \%)$ with a Xe lamp $(300 \mathrm{~W})$ and measuring the $\mathrm{H}_{2}$ produced at different time intervals. As shown in Fig. $5 \mathrm{c}$, MIL-100(Ti) shows an induction period of $c a .6 \mathrm{~h}$ for a total $\mathrm{H}_{2}$ production of $1000 \mu \mathrm{mol} \mathrm{g}_{\mathrm{cat}^{-1}}{ }^{-1}$ after 24 hours (see $\mathrm{S} 6 \dagger$ for experimental details). Stability was confirmed with PXRD and cyclability experiments for 5 consecutive cycles with minimum changes to the total $\mathrm{H}_{2}$ production measured (Fig. S40 and S41†).

The generation of photoinduced charge separation in MIL100(Ti), a hallmark feature of semiconductors, was confirmed with transient absorption spectroscopy (TAS). The decay kinetics profile of the solid suspended in ACN, measured at $500 \mathrm{~nm}$, shows two different processes. A first process that decays during the first $500 \mathrm{~ns}$, and a second one that remains stable in $\mathrm{Ar}$ or in the presence of $\mathrm{MeOH}$ for approximately $20 \mu \mathrm{s}$ after the light pulse (Fig. 5d). In addition, the longer-lived component is inhibited in the presence of $\mathrm{O}_{2}$ suggesting that it likely originates from photogenerated $\mathrm{Ti}(\mathrm{III})$ species in the framework. This is further confirmed by the TAS spectrum recorded at $60 \mathrm{~ns}$ after the light pulse, which shows an absorption band centred at $c a .640 \mathrm{~nm}$, characteristic of Ti(III) species (Fig. S42†). The intensity of this band decreases after 6 $\mu$ s showing a broad absorption between 400 and $600 \mathrm{~nm}$, likely due to the decay of an important fraction of the photogenerated species.

\section{Synthesis from other metal precursors}

Previous studies confirm the importance of choosing suitable titanium precursors for directing the synthesis of crystalline titanium frameworks. We are confident that the use of highthroughput synthetic methodologies might help in studying this reactivity more systematically in order to enlarge the pool of precursors at hand. This pushed us to investigate the synthesis of MIL-100(Ti) by using other Ti salts with variable reactivity in water. We replaced $\mathrm{Ti}_{6}$ with precursors like $\mathrm{Ti}\left(\mathrm{O}^{\mathrm{i}} \mathrm{Pr}\right)_{4}, \mathrm{Cp}_{2} \mathrm{TiCl}_{2}$ or $\mathrm{CpTiCl}_{3}$ and introduced minor changes to the synthesis optimized for the cluster. Our results confirm that MIL-100(Ti) can be also prepared from organometallic precursors. However, $\mathrm{Ti}\left(\mathrm{O}^{\mathrm{i}} \mathrm{Pr}\right)_{4}$ only afforded poorly crystalline solids for all the conditions explored (Fig. 2d), PXRD LeBail refinement was used to confirm the phase purity of the solids formed with $\mathrm{Cp}_{2} \mathrm{TiCl}_{2}$ and $\mathrm{CpTiCl}_{3}$. Full-profile fits yield excellent agreement factors and profile differences for both solids (Fig. S17 and S18†). SEM analysis also confirms the formation of micrometric particles of sizes below $1 \mu \mathrm{m}$ for $\mathrm{Cp}_{2} \mathrm{TiCl}_{2}$ and $\mathrm{CpTiCl}_{3}$ compared to the amorphous solid with ill-defined morphologies prepared with $\mathrm{Ti}\left(\mathrm{O}^{\mathrm{i}} \mathrm{Pr}\right)_{4}$ (Fig. 2f-h, S12 and S13 $)$. Our experiments suggest that organometallic precursors are more adequate than the isopropoxide to induce the formation of crystalline MIL-100(Ti), likely due to their higher resistance against hydrolysis.

For a better understanding of the impact of the metal precursor over the properties of the MOF, we next analysed the porosity of MIL-100(Ti)-Cp $\mathrm{Cp}_{2} \mathrm{TiCl}_{2}$ with $\mathrm{N}_{2}$ sorption experiments at $77 \mathrm{~K}$. Compared to MIL-100(Ti)-Ti ${ }_{6}$, the solid displays a similar isotherm and pore size distribution - indicative of the formation of the same open framework - but a substantial decrease in the BET surface area and pore volume to $1130 \mathrm{~m}^{2} \mathrm{~g}^{-1}$ and $0.51 \mathrm{~cm}^{3}$ $\mathrm{g}^{-1}$ (Fig. S23 and Table S3†). We argued this could be linked to the presence of bulkier $\mathrm{Cp}$ units replacing $\mathrm{OH}^{-}$as capping ligands for a concomitant decrease in porosity. The ability of cyclopentadienyl ligands to induce the formation of Cp-capped titanium clusters was first anticipated for COK-69. ${ }^{20}$ To confirm this point we carried out FT-IR, ${ }^{1} \mathrm{H}$-NMR and ${ }^{13} \mathrm{C}$-CP-MAS-NMR studies of the solid after acid digestion (Fig. S20, S27 and $\mathrm{S} 29 \dot{\dagger})$. All experiments discard the incorporation of $\mathrm{Cp}$ units into the material for the synthetic conditions used. To further confirm this point, we theoretically calculated the formation energy of the cluster $\left[\mathrm{Ti}_{3}\left(\mu_{3}-\mathrm{O}\right) \mathrm{O}(\mathrm{Cp})_{2}(\mathrm{btc})_{6}\right]$ (SI5). As shown in Fig. S38, $\dagger$ our calculations suggest that the incorporation of $\mathrm{Cp}$ linkers in axial positions in the Ti sites is thermodynamically disfavoured, for a difference in energy of 1.1 or $1.3 \mathrm{eV}$ over the formation of $\mathrm{OH}^{-} /$ $\mathrm{O}^{2-}$ or $\mathrm{O}^{2-} / \mathrm{H}_{2} \mathrm{O}$ capped clusters.

This systematic exploration of conditions and metal precursors suggests that the formation of trimeric $\mathrm{Ti}_{3}\left(\mu_{3}-\mathrm{O}\right)$ clusters units for the formation of MIL-100(Ti) under optimized reaction conditions is thermodynamically favoured not only for pre-formed clusters but also for organometallic complexes. However, the latter are less effective in preventing the formation of amorphous fractions of the material responsible for the observed reduction in porosity in MIL-100(Ti)- $\mathrm{Cp}_{2} \mathrm{TiCl}_{2}$. Overall, our experiments highlight the importance of controlling the 
reactivity of Ti precursors with water to enable the formation of crystalline, porous titanium-frameworks.

\section{Conclusions}

The design of new titanium-frameworks is arguably limited by the high reactivity of $\mathrm{Ti}(\mathrm{Iv})$ with water and the subsequent difficulties in controlling the formation of persistent SBUs with the rigidity and directionality required to target specific architectures. We have shown how the use of high-throughput synthetic methodologies is useful to identify the conditions required to form $\mathrm{Ti}_{3}\left(\mu_{3}-\mathrm{O}\right)$ metal-oxo clusters from different titanium precursors. This classical SBU can be then reticulated into a MIL-100 topology by direct reaction with trimesic acid to produce a mesoporous material with excellent thermal and hydrolytic stability, linked to the incorporation of highly charged Ti(Iv) atoms. MIL-100(Ti) also inherits the photoactivity of other titanium-frameworks for the generation of reactive Ti(III) species with light upon LMCT. In this regard, the combination of chemical stability and photoactivity into a mesoporous framework, also amenable to the generation of vacant sites, offers a promising route for the design of advanced porous photocatalysts without the metric limitations imposed by the microporosity of the materials currently available. Based on the preliminary results, we are confident that this MOF will also be compatible with linker functionalization or metal doping for engineering its optical response with visible light. In the context of biomedical applications, the low-toxicity of titanium and the different degradation rates compared to MIL100(Ti) might also be relevant for approaching targeted release of drugs.

\section{Experimental section}

\section{Chemical and physical characterization}

Infrared spectra were recorded on an Agilent Cary 630 FTIR spectrometer equipped with an ATR module. Thermogravimetric analysis was carried out with a Mettler Toledo TGA/SDTA $851 \mathrm{e} / \mathrm{SF} / 1100$ between 25 and $800{ }^{\circ} \mathrm{C}$ at a rate of $5^{\circ} \mathrm{C} \mathrm{min}^{-1}$ with a flow of $\mathrm{N}_{2}: \mathrm{O}_{2}(4: 1)$. PXRD patterns were collected in a PANalytical X'Pert PRO diffractometer using copper radiation $(\mathrm{Cu}$ $\mathrm{K}_{\alpha}$ ) with an X'Celerator detector, operating at $40 \mathrm{~mA}$ and $45 \mathrm{kV}$. Profiles were collected in the $2^{\circ}<2 \theta<90^{\circ}$ range with a step size of 0.013. Particle morphologies and dimensions were studied with a Hitachi S-4800 scanning electron microscope operating at $20 \mathrm{kV}$ over metalized samples. Surface area, pore size and volume values were calculated from nitrogen adsorptiondesorption isotherms recorded at $77 \mathrm{~K}$ on a Micromeritics 3Flex apparatus. Samples were degassed overnight at $150^{\circ} \mathrm{C}$ and $10^{-6}$ Torr prior to analysis. Surface areas were estimated according to the BET model and pore size dimensions were calculated using Non-Linear Density Functional Theory (NLDFT) models for the adsorption branch assuming a cylindrical pore model. ${ }^{13} \mathrm{C}-\mathrm{CP}-$ MAS-NMR was carried out on a Bruker Avance III $400 \mathrm{WB}$ Spectrometer. Samples were loaded in a $4 \mathrm{~mm}$ zirconia rotor and spun at $8 \mathrm{kHz} .{ }^{1} \mathrm{H}-\mathrm{NMR}$ spectra were run on a Bruker
DRX300 spectrometer. See ESI $\dagger$ for additional experimental details on digestion of the solids for NMR study.

\section{Synthesis of $\left[\mathrm{Ti}_{6} \mathrm{O}_{6}\left(\mathrm{O}^{\mathrm{i}} \mathrm{Pr}\right)_{6}(4-\mathrm{tbbz})_{6}\right]\left(\mathrm{Ti}_{6}\right)$}

The synthesis of the $\mathrm{Ti}_{6}$ cluster was carried out according to a previously reported procedure. ${ }^{47}$

\section{Synthesis of MIL-100(Ti)}

A typical synthesis of MIL-100(Ti) was carried out by adding $7.2 \mathrm{mg}$ of $\mathrm{Ti}_{6}(24 \mu \mathrm{mol}$ of $\mathrm{Ti})$ and $25.0 \mathrm{mg}$ of $\mathrm{H}_{3} \mathrm{btc}(120 \mu \mathrm{mol})$ to $3 \mathrm{~mL}$ of a mixture of ACN : THF $(3: 1, \mathrm{v} / \mathrm{v} \%)$ in a glass vial. Subsequently, $250 \mu \mathrm{L}$ of acetic acid (180 equiv.) were added and the mixture was sonicated to get a homogeneous suspension. The vial was placed in the oven at $160{ }^{\circ} \mathrm{C}$ for 48 hours. After cooling down to room temperature, the white microcrystalline powder was recovered by centrifugation, rinsed with fresh DMF and $\mathrm{MeOH}$ and further washed by Soxhlet extraction with hot $\mathrm{EtOH}$ or $\mathrm{MeOH}$ for several hours. The solid was then allowed to dry under vacuum at room temperature.

\section{Synthesis of MIL-100(Ti) from other Ti(Iv) precursors $\left(\mathrm{Cp}_{2} \mathbf{T i C l}_{2}, \mathrm{CpTiCl}_{3}\right.$, and $\left.\mathrm{Ti}\left(\mathrm{O}^{\mathrm{i}} \mathrm{Pr}\right)_{4}\right)$}

The synthesis of MIL-100(Ti) from simple Ti(Iv) precursors was carried out by adding $6.2 \mathrm{mg}$ of $\mathrm{Cp}_{2} \mathrm{TiCl}_{2}, 5.4 \mathrm{mg}$ of $\mathrm{CpTiCl}_{3}$ or $7.2 \mu \mathrm{L}$ of $\mathrm{Ti}\left(\mathrm{O}^{\mathrm{i}} \mathrm{Pr}\right)_{4}(24 \mu \mathrm{mol}$ of $\mathrm{Ti}(\mathrm{rv}))$, and $25.0 \mathrm{mg}$ of $\mathrm{H}_{3} \mathrm{btc}(120$ $\mu \mathrm{mol})$ to $3 \mathrm{~mL}$ of a mixture of ACN : THF $(3: 1, \mathrm{v} / \mathrm{v} \%)$ in a glass vial. Subsequently, $500 \mu \mathrm{L}$ of acetic acid were added and the mixture was sonicated to get a homogeneous suspension. The vial was placed in the oven at $160{ }^{\circ} \mathrm{C}$ for 12 hours. After cooling down to room temperature, the white microcrystalline powder was recovered by centrifugation, rinsed with fresh DMF and $\mathrm{MeOH}$ and further washed by Soxhlet extraction with hot EtOH or $\mathrm{MeOH}$ for several hours. The solid was then allowed to dry under vacuum at room temperature.

\section{Conflicts of interest}

There are no conflicts to declare.

\section{Acknowledgements}

This work was supported by the EU (ERC Stg Chem-fs-MOF 714122) and Spanish MINECO (MDM-2015-0538 \& CTQ201783486-P). C. M.-G. and J. C.-G. thank the Spanish MINECO for a Ramón y Cajal Fellowship (RYC-2012-10894) and FPI Scholarship (CTQ2014-59209-P). N. M. P. thanks the European Union for a Marie Skłodowska-Curie Global Fellowship (H2020-MSCAIF-2016-GF-749359-EnanSET). We thank the ISIS Facility for the access to synchrotron radiation, BSC-RES and Prof. Enrique Ortí for computational resources and Belén Lerma-Berlanga for technical help.

\section{Notes and references}

1 H. Furukawa, K. E. Cordova, M. O'Keeffe and O. M. Yaghi, Science, 2013, 341, 1230444. 
2 R. V. Jagadeesh, K. Murugesan, A. S. Alshammari, H. Neumann, M.-M. Pohl, J. Radnik and M. Beller, Science, 2017, 358, 326-332.

3 A. Cadiau, Y. Belmabkhout, K. Adil, P. M. Bhatt, R. S. Pillai, A. Shkurenko, C. Martineau-Corcos, G. Maurin and M. Eddaoudi, Science, 2017, 356, 731-735.

4 H. Kim, S. Yang, S. R. Rao, S. Narayanan, E. A. Kapustin, H. Furukawa, A. S. Umans, O. M. Yaghi and E. N. Wang, Science, 2017, 356, 430-434.

5 D. Sheberla, J. C. Bachman, J. S. Elias, C.-J. Sun, Y. Shao-Horn and M. Dincă, Nat. Mater., 2017, 16, 220-224.

6 J. E. Mondloch, M. J. Katz, W. C. Isley, P. Ghosh, P. Liao, W. Bury, G. W. Wagner, M. G. Hall, J. B. Decoste, G. W. Peterson, R. Q. Snurr, C. J. Cramer, J. T. Hupp and O. K. Farha, Nat. Mater., 2015, 14, 512-516.

7 A. J. Howarth, Y. Liu, P. Li, Z. Li, T. C. Wang, J. T. Hupp and O. K. Farha, Nat. Rev. Mater., 2016, 1, 15018.

8 K. Park, Z. Ni, A. Côté, J. Choi, R. Huang, F. Uribe-Romo, H. Chae, M. O'Keeffe and O. M. Yaghi, Proc. Natl. Acad. Sci. U. S. A., 2006, 103, 10186.

9 V. Colombo, S. Galli, H. J. Choi, G. D. Han, A. Maspero, G. Palmisano, N. Masciocchi and J. R. Long, Chem. Sci., 2011, 2, 1311.

10 H. Assi, G. Mouchaham, N. Steunou, T. Devic and C. Serre, Chem. Soc. Rev., 2017, 46, 3431-3452.

11 J. Cavka, S. Jakobsen, U. Olsbye, N. Guillou, C. Lamberti, S. Bordiga and K. Lillerud, J. Am. Chem. Soc., 2008, 130, 13850-13851.

12 Y. Bai, Y. Dou, L.-H. Xie, W. Rutledge, J.-R. Li and H.-C. Zhou, Chem. Soc. Rev., 2016, 45, 2327-2367.

13 T. Devic and C. Serre, Chem. Soc. Rev., 2014, 43, 6097-6115. 14 M. Kim and S. M. Cohen, CrystEngComm, 2012, 14, 40964104.

15 S. Wang, T. Kitao, N. Guillou, M. Wahiduzzaman, C. Martineau-Corcos, F. Nouar, A. Tissot, L. Binet, N. Ramsahye, S. Devautour-Vinot, S. Kitagawa, S. Seki, Y. Tsutsui, V. Briois, N. Steunou, G. Maurin, T. Uemura and C. Serre, Nat. Commun., 2018, 9, 1660.

16 M. Dan-Hardi, C. Serre, T. Frot, L. Rozes, G. Maurin, C. Sanchez and G. Ferey, J. Am. Chem. Soc., 2009, 131, 10857-10859.

17 S. Yuan, T.-F. Liu, D. Feng, J. Tian, K. Wang, J. qin, Q. Zhang, Y.-P. Chen, M. Bosch, L. Zou, S. J. Teat, S. J. Dalgarno and H.-C. Zhou, Chem. Sci., 2015, 6, 3926-3930.

18 H. L. Nguyen, F. Gándara, H. Furukawa, T. L. H. Doan, K. E. Cordova and O. M. Yaghi, J. Am. Chem. Soc., 2016, 138, 4330-4333.

19 C. Wang, C. Liu, X. He and Z.-M. Sun, Chem. Commun., 2017, 53, 11670-11673.

20 B. Bueken, F. Vermoortele, D. E. P. Vanpoucke, H. Reinsch, C.-C. Tsou, P. Valvekens, T. De Baerdemaeker, R. Ameloot, C. E. A. Kirschhock, V. Van Speybroeck, J. M. Mayer and D. De Vos, Angew. Chem., Int. Ed., 2015, 54, 13912-13917.

21 S. Yuan, J.-S. Qin, H.-Q. Xu, J. Su, D. Rossi, Y. Chen, L. Zhang, C. Lollar, Q. Wang, H.-L. Jiang, D. H. Son, H. Xu, Z. Huang, X. Zou and H.-C. Zhou, ACS Cent. Sci., 2018, 4, 105-111.
22 J. Castells-Gil, N. M. Padial, N. Almora Barrios, J. Albero, A. R. Ruiz-Salvador, J. Gonzalez-Platas, H. García and C. Martí-Gastaldo, Angew. Chem., Int. Ed., 2018, 57, 84538457.

23 J. Gao, J. Miao, P.-Z. Li, W. Y. Teng, L. Yang, Y. Zhao, B. Liu and Q. Zhang, Chem. Commun., 2014, 50, 3786-3794.

24 N. T. T. Nguyen, H. Furukawa, F. Gándara, C. A. Trickett, H. M. Jeong, K. E. Cordova and O. M. Yaghi, J. Am. Chem. Soc., 2015, 137, 15394-15397.

25 M. Kim, J. F. Cahill, H. Fei, K. A. Prather and S. M. Cohen, J. Am. Chem. Soc., 2012, 134, 18082-18088.

26 C. K. Brozek and M. Dincă, J. Am. Chem. Soc., 2013, 135, 12886-12891.

27 L. Zou, D. Feng, T.-F. Liu, Y.-P. Chen, S. Yuan, K. Wang, X. Wang, S. Fordham and H.-C. Zhou, Chem. Sci., 2016, 7, 1063-1069.

28 J. G. Santaclara, A. I. Olivos-Suarez, A. Gonzalez Nelson, D. Osadchii, M. A. Nasalevich, M. A. van der Veen, F. Kapteijn, A. M. Sheveleva, S. L. Veber, M. V. Fedin, A. T. Murray, C. H. Hendon, A. Walsh and J. Gascon, Chem. Mater., 2017, 29, 8963-8967.

29 M. S. Denny, L. R. Parent, J. P. Patterson, S. K. Meena, H. Pham, P. Abellan, Q. M. Ramasse, F. Paesani, N. C. Gianneschi and S. M. Cohen, J. Am. Chem. Soc., 2018, 140, 1348-1357.

30 V. Guillerm, S. Gross, C. Serre, T. Devic, M. Bauer and G. Ferey, Chem. Commun., 2010, 46, 767-769.

31 S. Smolders, A. Struyf, H. Reinsch, B. Bueken, T. Rhauderwiek, L. Mintrop, P. Kurz, N. Stock and D. E. De Vos, Chem. Commun., 2018, 112, 869.

32 D. Feng, K. Wang, Z. Wei, Y.-P. Chen, C. M. Simon, R. K. Arvapally, R. L. Martin, M. Bosch, T.-F. Liu, S. Fordham, D. Yuan, M. A. Omary, M. Haranczyk, B. Smit and H.-C. Zhou, Nat. Commun., 2014, 5, 5723-5729.

33 G. Ferey, C. Serre, C. Mellot-Draznieks, F. Millange, S. Surblé, J. Dutour and I. Margiolaki, Angew. Chem., Int. Ed., 2004, 43, 6296-6301.

34 P. Horcajada, S. Surblé, C. Serre, D.-Y. Hong, Y.-K. Seo, J.-S. Chang, J.-M. Grenèche, I. Margiolaki and G. Ferey, Chem. Commun., 2007, 2820-2822.

35 Y. Keum, S. Park, Y.-P. Chen and J. Park, Angew. Chem., Int. Ed., 2018, 238, 37.

36 T. Loiseau, L. Lecroq, C. Volkringer, J. Marrot, G. Ferey, M. Haouas, F. Taulelle, S. Bourrelly, P. L. Llewellyn and M. Latroche, J. Am. Chem. Soc., 2006, 128, 10223-10230.

37 G. Ferey, C. Mellot-Draznieks, C. Serre, F. Millange, J. Dutour, S. Surblé and I. Margiolaki, Science, 2005, 309, 2040-2042.

38 A. Sonnauer, F. Hoffmann, M. Fröba, L. Kienle, V. Duppel, M. Thommes, C. Serre, G. Ferey and N. Stock, Angew. Chem., Int. Ed., 2009, 48, 3791-3794.

39 J. W. Yoon, Y.-K. Seo, Y. K. Hwang, J.-S. Chang, H. Leclerc, S. Wuttke, P. Bazin, A. Vimont, M. Daturi, E. Bloch, P. L. Llewellyn, C. Serre, P. Horcajada, J.-M. Grenèche, A. E. Rodrigues and G. Ferey, Angew. Chem., Int. Ed., 2010, 49, 5949-5952. 
40 M. J. Kalmutzki, C. S. Diercks and O. M. Yaghi, Adv. Mater., 2018, 289, 1704304.

41 M. Wickenheisser, A. Herbst, R. Tannert, B. Milow and C. Janiak, Microporous Mesoporous Mater., 2015, 215, 143153.

42 F. Jeremias, A. Khutia, S. K. Henninger and C. Janiak, J. Mater. Chem., 2012, 22, 10148-10151.

43 D. R. Lide, CRC Handbook of Chemistry and Physics, CRC Press, 85th edn, 2004, vol. 127.
44 P. Horcajada, R. Gref, T. Baati, P. K. Allan, G. Maurin, P. Couvreur, G. Ferey, R. E. Morris and C. Serre, Chem. Rev., 2012, 112, 1232-1268.

45 A. Dhakshinamoorthy, A. M. Asiri and H. García, Angew. Chem., Int. Ed., 2016, 55, 5414-5445.

46 C. H. Hendon, D. Tiana, M. Fontecave, C. Sanchez, L. D'arras, C. Sassoye, L. Rozes, C. Mellot-Draznieks and A. Walsh, J. Am. Chem. Soc., 2013, 135, 10942-10945.

47 K. Hong, W. Bak and H. Chun, Inorg. Chem., 2014, 53, 72887293. 